\title{
CCND1/CyclinD1 status in metastasizing bladder cancer: a prognosticator and predictor of chemotherapeutic response
}

\author{
Roland Seiler ${ }^{1,2}$, George N Thalmann ${ }^{2}$, Diana Rotzer ${ }^{1}$, Aurel Perren ${ }^{1}$ and \\ Achim Fleischmann ${ }^{1}$ \\ ${ }^{1}$ Institute of Pathology, University of Bern, Bern, Switzerland and ${ }^{2}$ Department of Urology, \\ University of Bern, Bern, Switzerland
}

\begin{abstract}
The CCND1 gene encodes the protein CyclinD1, which is an important promoter of the cell cycle and a prognostic and predictive factor in different cancers. CCND1 is amplified to a substantial proportion in various tumors, and this may contribute to CyclinD1 overexpression. In bladder cancer, information about the clinical relevance of CCND1/CyclinD1 alterations is limited. In the present study, amplification status of CCND1 and expression of CyclinD1 were evaluated by fluorescence in situ hybridization and immunohistochemistry on tissue microarrays from 152 lymph node-positive urothelial bladder cancers (one sample each from the center and invasion front of the primary tumors, two samples per corresponding lymph node metastasis) treated by cystectomy and lymphadenectomy. CCND1 amplification status and the percentage of immunostained cancer cells were correlated with histopathological tumor characteristics, cancer-specific survival and response to adjuvant chemotherapy. CCND1 amplification in primary tumors was homogeneous in $15 \%$ and heterogeneous in 6\% (metastases: 22 and 2\%). Median nuclear CyclinD1 expression in amplified samples was similar in all tumor compartments $(60-70 \%$ immunostained tumor nuclei) and significantly higher than in non-amplified samples $(5-20 \%$ immunostained tumor nuclei; $\boldsymbol{P}<0.05)$. CCND1 status and CyclinD1 expression were not associated with primary tumor stage or lymph node tumor burden. CCND1 amplification in primary tumors $(P=0.001)$ and metastases $(P=0.02)$ and high nuclear CyclinD1 in metastases $(P=0.01)$ predicted early cancerrelated death independently. Subgroup analyses showed that chemotherapy was particularly beneficial in patients with high nuclear CyclinD1 expression in the metastases, whereas expression in primary tumors and CCND1 status did not predict chemotherapeutic response. In conclusion, CCND1 amplification status and CyclinD1 expression are independent risk factors in metastasizing bladder cancer. High nuclear CyclinD1 expression in lymph node metastases predicts favorable response to chemotherapy. This information may help to personalize prognostication and administration of adjuvant chemotherapy.
\end{abstract}

Modern Pathology (2014) 27, 87-95; doi:10.1038/modpathol.2013.125; published online 26 July 2013

Keywords: bladder cancer; CCND1; CyclinD1 chemotherapy response prediction; survival

Approximately $25 \%$ of clinically staged N0M0 and surgically treated bladder cancer patients have lymph node metastases upon histological evaluation. ${ }^{1,2}$ These patients are at high risk for disease recurrence and about two-thirds will die from cancer. $^{2,3}$ Better prediction of their clinical course and response to adjuvant therapies is necessary to improve patient management.

Correspondence: Professor A Fleischmann, MD, Institute of Pathology, University of Bern, Murtenstrasse, Bern CH-3010, Switzerland.

E-mail: achim.fleischmann@pathology.unibe.ch

Received 29 January 2013; revised 17 May 2013; accepted 17 May 2013; published online 26 July 2013
Aberrant expression of the protein CyclinD1 and alterations in its coding gene CCND1 are frequent in human cancers ${ }^{4}$ and may harbor prognostic ${ }^{5-9}$ and predictive $^{10-12}$ information. CyclinD1 is primarily known as a key regulator of the cell cycle. It forms complexes with cyclin-dependent kinase 4 or 6 in the cytoplasm, these complexes then enter the nucleus ${ }^{4}$ and inactivate the cell-cycle suppressive retinoblastoma protein, thereby promoting progression from $\mathrm{G} 1$ to the S-phase. CyclinD1 expression is physiologically controlled by different signal transduction pathways. ${ }^{13}$ In cancer, CyclinD1 is frequently overexpressed. Different mechanisms may drive this process, including upregulation of transduction pathways and CCND1 
amplification. ${ }^{14-16}$ However, amplification does not necessarily leads to protein overexpression, suggesting complex expression mechanisms. ${ }^{17-19}$

In bladder cancer, information on frequency of CCND1/CyclinD1 alterations and their clinical relevance are limited. The prognostic impact has primarily been evaluated in non-muscle invasive bladder cancer for CyclinD1 and with regard to recurrence- and progression-free survival. However, data were inconsistent showing CyclinD1 expression as a marker of adverse ${ }^{20-22}$ or favorable ${ }^{23-27}$ prognosis or without prognostic impact. ${ }^{28}$ Only two studies investigated the prognostic relevance of CCND1 amplification in bladder cancer: while CCND1 amplification was an adverse risk factor in non-muscle invasive bladder cancers, ${ }^{29}$ it failed to predict survival in muscle invasive tumors. ${ }^{30}$ Finally, CyclinD1 expression has been shown to predict response to chemotherapies in cancers of the head and neck; ${ }^{10,11}$ however, this potential has never been tested in bladder cancer. We evaluated these open questions on CCND1/CyclinD1 status in a homogeneous cohort of lymph node-positive bladder cancer patients treated by cystectomy and extended lymphadenectomy as well as adjuvant chemotherapy in a subset of patients.

\section{Materials and methods}

\section{Patient Selection}

Bladder cancer patients $(n=152)$ preoperatively staged cN0cM0 (physical examination, chest X-ray, abdominal and pelvic computerized tomography and bone scan) but lymph node metastases upon pathological examination were enrolled for the study. A standardized extended pelvic lymphadenectomy with cystectomy was performed between January 1985 and April 2008 at the Department of Urology, University of Bern, Bern, Switzerland. No patient received neoadjuvant therapy.

\section{Follow-Up and Adjuvant Chemotherapy}

Patients were followed prospectively according to a standard protocol as follows: In general, clinical evaluation, blood examination and ultrasound were performed postoperatively at 3 and 6 months, then at 6-month intervals until 5 years after cystectomy and yearly thereafter. Additionally, an abdominal and pelvic computerized tomography was performed at 6 and 12 months postoperatively when becoming available. In case of aggressive disease (eg, high number of positive lymph nodes, presence of extranodal extension (ENE)) or recurrence, the recommendation for adjuvant chemotherapy was made after a multidisciplinary discussion. Chemotherapy was given in $41 \%(63 / 152)$. A platinbased regimen was administered in $59 \%(37 / 63)$ of the patients; the others $(n=26)$ received Vincristine,
Methotrexat, Leucovorin, Navelbine or Vinflunine in various combinations for different health reasons.

\section{Surgical Technique and Pathology}

Bilateral pelvic lymphadenectomy was performed in all the patients according to a standard protocol. ${ }^{31}$ All lymphatic tissues were meticulously removed from the common iliac bifurcation along the external iliac vessels to the inguinal ligament, from the obturator fossa and from the ventral aspect of the internal iliac vein as well as the internal iliac artery and branches. The cystectomy protocol has previously been described in detail. ${ }^{32}$

The opened bladder and lymphadenectomy specimens were fixed overnight in neutral-buffered formalin and processed at the Institute of Pathology, University of Bern, Bern, Switzerland. Samples taken from the bladder included the deepest macroscopic invasion of the tumor. Microscopically, tumor stage and grade were determined. The lymphadenectomy specimens from each anatomical region were examined by inspection and palpation. All macroscopically detected lymph nodes were embedded completely. Whenever no identification of nodes was possible, the entire tissue was embedded for histological examination. All tumors were staged according to the seventh International Union Against Cancer classification of 2009. ${ }^{33}$

\section{Construction of Tissue Microarray}

Tissue microarray ${ }^{34}$ was constructed with four samples (diameter: $0.6 \mathrm{~mm}$ ) per patient, two from primary tumors (center and invasion front) and two from a corresponding lymph node metastases (samples A and B).

\section{Fluorescence In Situ Hybridization (FISH) and Immunohistochemistry (IHC)}

Two consecutive sections of $3-\mu \mathrm{m}$ thickness were taken for FISH and IHC assays.

For FISH analysis of CCND1 status, the Vysis CCND1 /CEP 11 FISH Probe Kit was used (Vysis, Downers Grove, IL). It is a mixture of LSI CCND1 gene (11q13) labelled in SpectrumOrange and CEP11 SpectrumGreen, directed against the centromeric satellite repeat of chromosome 11. Detailed instructions for hybridization procedures are provided by the manufacturer. Briefly, tissue array sections (3- $\mu$ m thick) were dewaxed and rehydrated in graded ethanol. After pre-treatment, tissues were denatured for $5 \mathrm{~min}$ at $73{ }^{\circ} \mathrm{C}$ in a $70 \%$ formamide/ 2X-SSC solution. Samples were then dehydrated and subsequently treated with proteinase K. The hybridization mixture contained $1 \mu \mathrm{l}$ LSI probe diluted in the hybridization mix. After overnight hybridization at $37^{\circ} \mathrm{C}$, slides were washed and 

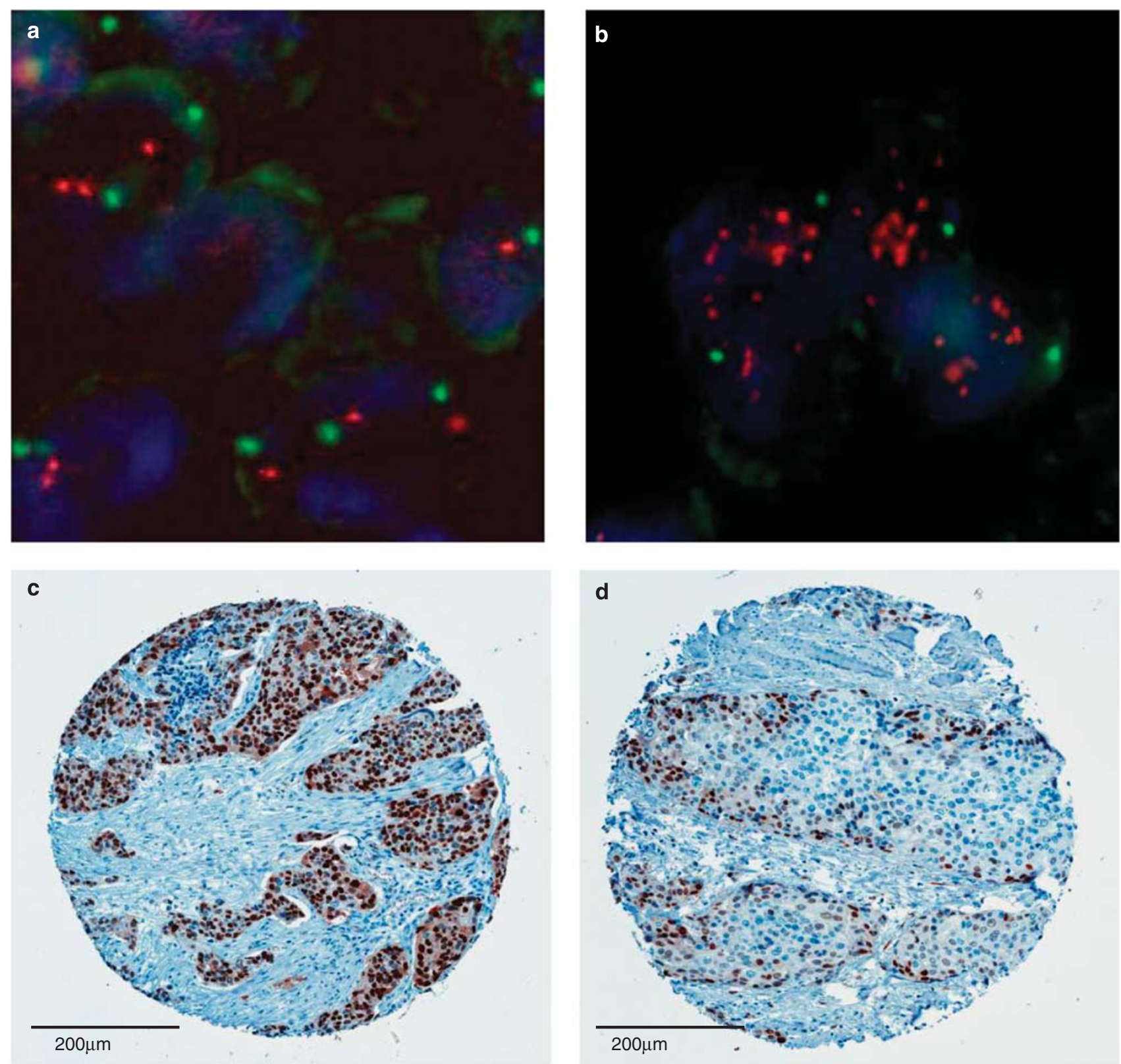

Figure 1 Bladder cancer samples without CCND1 amplification (a) show a gene/centromer 11 (red signals/green signals) ratio <2 and those with amplification (b) a ratio $\geq 2$. Immunostains of tumors with high (c; $>80 \%$ positive cancer nuclei) and low (d) nuclear CyclinD1 expression.

counterstained with DAPI II (125 ng/ml, Vysis) in an antifading solution. A minimum of 25 non-overlapping nuclei were evaluated on each spot, and the individual spot was considered normal or amplified according to the CCND1/CEP11 fluorescence ratios $<2$ or $\geq 2^{18}$ (Figures 1a and b).

For IHC staining, before incubation slides were dewaxed, rehydrated and boiled in $1 \mathrm{mM}$ EDTA$10 \mathrm{mM}$ Tris, pH 9.0 in a microwave oven. CyclinD1 expression was assessed using a monoclonal rabbit antibody (clone SP4; Thermo Fisher Scientific, Kalamazoo, MI, USA). Optimal staining was achieved at 1:25 antibody dilution. Bound primary antibodies were visualized using the Envision Plus system (Dako, Glostrup, Denmark). CyclinD1 expression was observed in the nuclei and cytoplasm $^{27}$ and evaluated separately for percentage of positive tumor cells (Figures 1c and d).

A summary of the number of samples that were available for analysis is shown in Figure 2.

\section{Statistical Analyses}

For each primary tumor and each corresponding lymph node metastasis, (a) a CCND1 status based on the presence (homogeneous or heterogeneous) or absence of amplification and (b) a CyclinD1 

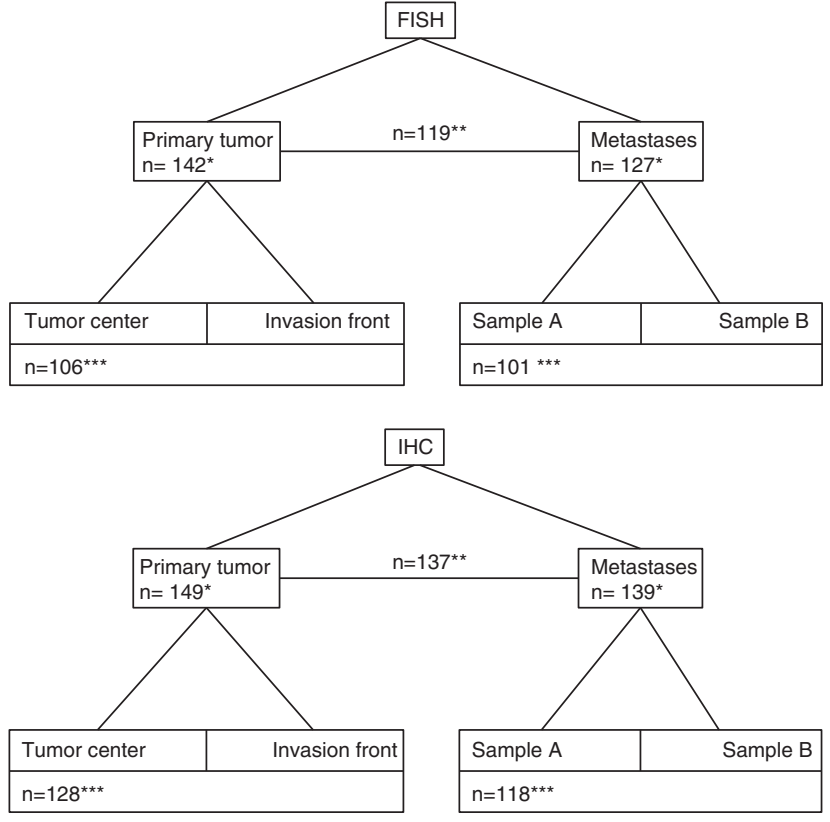

* At least one informative sample per tumor component.

* Corresponding samples from both tumor components.

*** Two informative samples in a given tumor component.

Figure 2 Flow chart summarizing the number of samples that are included in a given analysis.

expression level (nuclear, cytoplasmic) based on the sample with the highest percentage of immunostained tumor cells was assigned. These parameters were used to compare primary tumors with metastases and for correlations with histopathological tumor features (primary tumor stage; ENE and number of lymph node metastases; two-sided Wilcoxon's rank-sum test for non-categorical data and Fisher's test for categorical data) and cancer-specific survival (CSS). Concordance in FISH results between (a) the two samples of a primary tumor, (b) the two samples of a lymph node metastasis and (c) a primary tumor and its lymph node metastasis was evaluated with Cohen's kappa test; corresponding investigations for CyclinD1 expression used Pearson's correlation. Kaplan-Meier plots were used to estimate CSS from surgery to the date of cancer-related death (data available for 148 patients). This end point was chosen, because qualification for adjuvant chemotherapy might already be a feature with impact on overall survival that would bias such analyses. Patients still living and those who died without evidence of tumor recurrence were censored at the date of last follow-up and death, respectively. Differences in CSS between the subgroups defined according to CCND1 status (nonamplified vs amplified) and CyclinD1 expression level were assessed using the log-rank test. Survival according to the expression level was analyzed in quartiles. Metastases but not primary tumors showed similar outcome in the first three quartiles, which were therefore clustered (low expression) and compared with the fourth quartile (high expression;
Table 1 Clinico-pathological data of 152 lymph node-positive patients with urothelial cancer of the bladder

\begin{tabular}{lc} 
Patient data $(\mathrm{n}=152)$ & \\
\hline Age at surgery (years), median (range) & $67(35-89)$ \\
Female/male (n) & $29 / 123$ \\
Follow-up (years), median & 7.2 \\
5-year cancer-specific survival & $37 \%$ \\
& \\
Cystectomy data & \\
Tumor stage ( $n$ ) & $4 / 17$ \\
$\quad$ pT1/2 & $93 / 38$ \\
pT3/4 & \\
& \\
Lymphadenectomy data & $27(10-56)$ \\
Evaluated nodes per patient (n), median (range) & $3(1-46)$ \\
Positive nodes per patient $(n)$, median (range) & \\
Lymph node stage $(n)$ & 44 \\
$\quad$ pN1 & 108 \\
pN2/3 & \\
Extranodal extension $(n)$ & 71 \\
$\quad$ No & 81 \\
$\quad$ Yes &
\end{tabular}

$>80 \%$ positive cancer nuclei) in both the tumor compartments. To identify independent prognostic factors, Cox proportional hazards models were applied. $P$-values $<0.05$ were considered significant. All statistical analyses were performed using the R software package, Version 2.9.1., http://www. r-project.org.

\section{Results}

Clinico-pathological data of the cohort are given in Table 1.

\section{CCND1 Amplification and CyclinD1 Expression in Primary Tumors and Lymph Node Metastases and Their Association with Tumor Characteristics}

CCND1 amplification status in the tumor center and at the invasion front was concordant in $94 \%$ of the 106 tested primary tumors with two informative samples $(15 \% \quad(n=16)$ amplified, $79 \% \quad(n=84)$ non-amplified) and discordant in $6 \%(n=6)$; concordance between samples $\mathrm{A}$ and $\mathrm{B}$ in the metastases $(n=101)$ was $97 \%(22 \%(n=22)$ amplified, $75 \%(n=76)$ non-amplified), discordance was $3 \%$ $(n=3)$. CCND1 amplification status of both the primary tumor and a corresponding metastasis could be tested in 119 patients and was highly concordant (Cohen's Kappa: 0.9). Only one of the 22 amplified primary tumors had no evidence for amplification in the metastasis, and four of the 25 patients with amplified metastases showed no amplification in their primary tumors.

Nuclear and cytoplasmic Cyclin D1 expressions were significantly higher in metastases (median immunostained cancer cells: 50 and 15\%) compared with primary tumors (30 and $0 \%, P<0.05$ ). Nuclear 


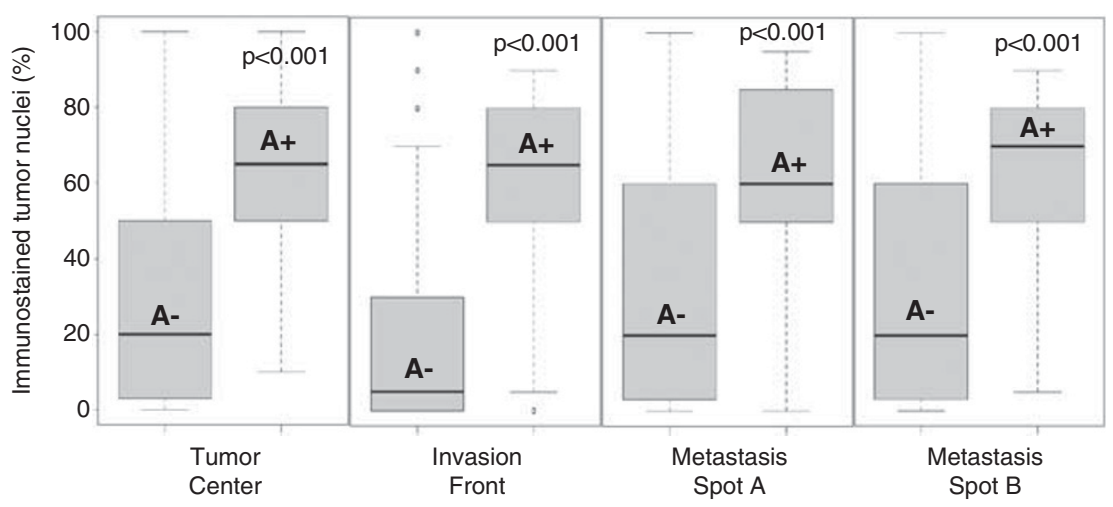

Figure 3 Box plots showing nuclear CyclinD1 expression in all the four tumor areas segregated for CCND1 non-amplified (A - )- and amplified $(\mathrm{A}+)$ tumor samples. In all the areas, median nuclear CyclinD1 expression in the amplified samples is similar and significantly higher than in the non-amplified samples, while the range of CyclinD1 expression is virtually independent of the amplification status.

expression in primary tumors and their metastases was well correlated (correlation coefficient (CC): 0.65), as was the expression in both samples of the primary tumors (CC: 0.72 ) and the metastases (CC: 0.78), respectively. Cytoplasmic expression in primary tumors and metastases was only weakly correlated (CC: 0.32), but the correlation between the two samples from the primary tumors (CC: 0.7) and from the metastases (CC:0.7) was good.

Median nuclear CyclinD1 expression level of the CCND1 amplified samples was similar in each region (median of immunostained tumor nuclei 60-70\%; Figure 3) and significantly $(P<0.05)$ higher than in non-amplified samples (5-20\% immunostained tumor nuclei). However, the range of CyclinD1 expression in the amplified and in non-amplified samples was the same $(0-100 \%$ immunostained tumor nuclei).

CCND1/CyclinD1 status in the primary tumors and in the metastases was not significantly related to the stage of the primary tumor or lymph nodes or to ENE of metastases. However, there was a trend $(P>0.1)$ for higher amplification rate $(22 \%)$ and median nuclear expression $(38 \%)$ in locally advanced pT3/4 compared with organ-confined pT1/2 (5 and 5\%) primary tumors.

\section{Univariate and Multivariate Survival Analysis}

For survival analyses, FISH data were available from 142 primary tumors and from 127 lymph node metastases with at least one informative tissue sample per tumor component (for IHC in 149 primary tumors and 139 metastases). CSS was significantly reduced when primary tumors $(P<0.001$, Figure 4a) or lymph node metastases $(P=0.02$, Figure 4b) were amplified and when nuclear CyclinD1 expression in metastases was high (expression $\geq 4$ th quartile, $P=0.001$, Figure $4 \mathrm{~d}$ ). These three prognosticators were independent from other known risk factors $(P=0.01, P=0.001$,
$P=0.02$, Table 2). High nuclear CyclinD1 expression in the primary tumors $(P=0.8$, Figure 4c) and cytoplasmic CyclinD1 in any tumor compartment were no prognosticators.

\section{CCND1/CyclinD1 Status and Adjuvant Chemotherapy}

Patients with high nuclear expression of CyclinD1 in their metastasizing tumor component had significantly different CSS depending on chemotherapy status: Virtually all patients without adjuvant chemotherapy died from cancer during the first 2.5 years after surgery (Figure 5a) while CSS significantly ameliorated when any chemotherapy (5-year CSS: $22 \%$, Figure 5b) and particularly platin-based chemotherapy (5-year CSS: 37\%, Figure 5c) was applied. No similar effects after adjuvant chemotherapy were observed in the subgroup of patients with low CyclinD1 expression in the metastases and also not in any other subgroup defined according to the amplification status or protein expression.

\section{Discussion}

The CCND1 gene and expression of its protein product CyclinD1 are frequently altered in human cancers and may predict tumor progression. ${ }^{35}$ However, in bladder cancer data on this wellestablished oncogene are still limited. Therefore, we investigated CCND1 amplification and CyclinD1 expression in a homogeneous cohort of lymph nodepositive bladder cancer patients with regard to tumor heterogeneity, correlation of amplification and protein expression as well as association with histopathological tumor characteristics, survival and response to chemotherapy.

Genomic instability is frequent in human cancers, which as a consequence harbor genetically diverse populations of tumor cells. ${ }^{36}$ This intra-tumor heterogeneity can influence disease progression and therapeutic response via cell subclones able to 
a

Primary tumors Cancer-specific survival

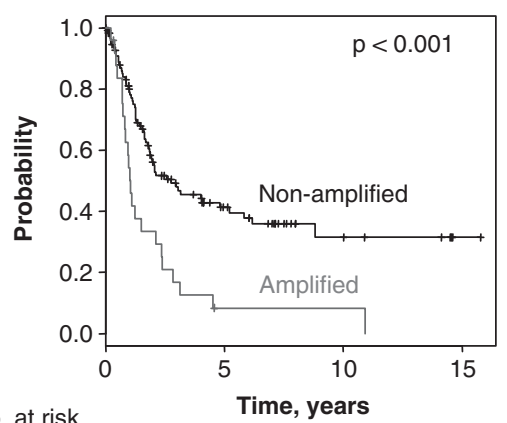

No. at risk

Non-amp. 114

Amplified 25

C

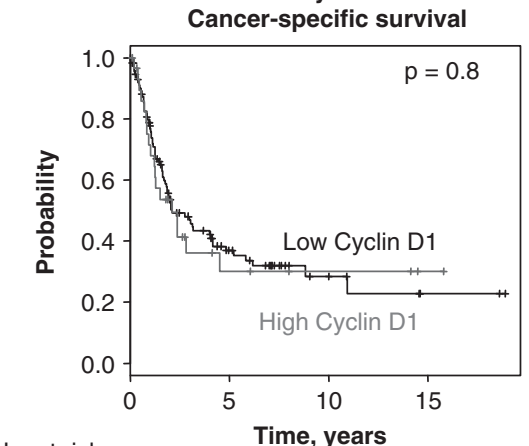

No. at risk

$\begin{array}{llll}\text { Non-amp. } 116 & 24 & 7 & 2\end{array}$

$\begin{array}{lllll}\text { Amplified } & 35 & 5 & 3 & 1\end{array}$ b

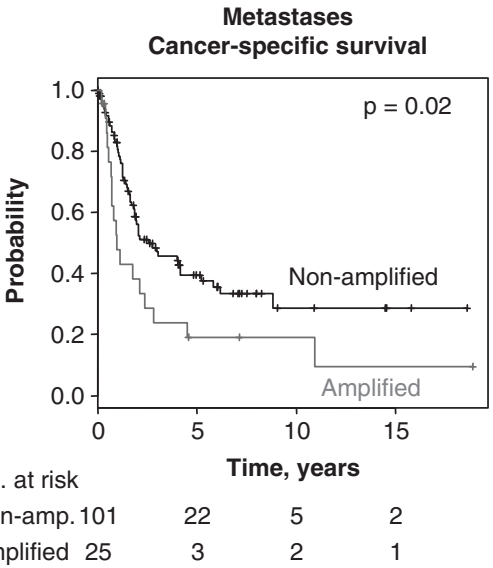

d

Metastases
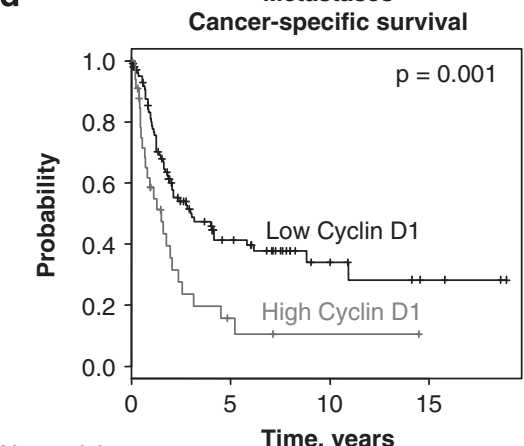

No. at risk

Non-amp. 103

Amplified 33

Figure 4 Cancer-specific survival stratified according to the CCND1 amplification status (a, b) and nuclear CyclinD1 expression $(<4$ th vs 4 th quartile; c, d) in primary tumors (a, c) and lymph node metastases (b, d).

Table 2 Multivariate analysis for cancer-specific survival: CCND1 amplification and high nuclear CyclinD1 expression in lymph node metastases predict early cancer-related death independently from other established risk factors

\begin{tabular}{|c|c|c|c|c|c|c|c|}
\hline \multirow{2}{*}{$\begin{array}{l}\text { Fluorescence } \\
\text { in situ hybridization }\end{array}$} & \multirow{2}{*}{$\begin{array}{l}\text { Localization } \\
\text { Primary tumor }\end{array}$} & \multirow{2}{*}{$\begin{array}{l}\text { Variable } \\
\text { Amplified }\end{array}$} & \multirow{2}{*}{$\begin{array}{l}\text { Reference level } \\
\text { Non-amplified }\end{array}$} & \multirow{2}{*}{$\begin{array}{l}H R \\
2.35\end{array}$} & \multicolumn{2}{|c|}{$95 \% C I$} & \multirow{2}{*}{$\begin{array}{c}\mathrm{P} \\
\mathbf{0 . 0 0 1}\end{array}$} \\
\hline & & & & & 1.44 & 3.83 & \\
\hline & & $\begin{array}{l}\text { Extranodal extension of } \\
\text { metastases }\end{array}$ & Without & 2.07 & 1.27 & 3.37 & 0.003 \\
\hline & & $\mathrm{pN} 2 / 3$ & pN1 & 1.41 & 0.78 & 2.57 & 0.26 \\
\hline & & $\mathrm{pT} 3 / 4$ & $\mathrm{pT} 1 / 2$ & 3.41 & 1.40 & 8.55 & 0.008 \\
\hline & Metastasis & Amplified & Non-amplified & 1.75 & 1.02 & 3.0 & 0.04 \\
\hline & & $\begin{array}{l}\text { Extranodal extension of } \\
\text { metastases }\end{array}$ & Without & 1.53 & 0.91 & 2.6 & 0.1 \\
\hline & & $\mathrm{pN} 2 / 3$ & pN1 & 1.62 & 0.8 & 3.3 & 0.18 \\
\hline & & $\mathrm{pT} 3 / 4$ & $\mathrm{pT} 1 / 2$ & 2.48 & 0.98 & 6.27 & 0.05 \\
\hline \multirow{8}{*}{$\begin{array}{l}\text { Immunohistochemistry } \\
\text { (nuclear) }\end{array}$} & Primary tumor & Expression $\geq 4$ th quartile & $\leq$ 3rd quartile & 1.37 & 0.8 & 2.32 & 0.25 \\
\hline & & $\begin{array}{l}\text { Extranodal extension of } \\
\text { metastases }\end{array}$ & Without & 1.94 & 1.2 & 3.16 & 0.007 \\
\hline & & $\mathrm{pN} 2 / 3$ & pN1 & 1.5 & 0.82 & 2.74 & 0.19 \\
\hline & & $\mathrm{pT} 3 / 4$ & $\mathrm{pT} 1 / 2$ & 3.03 & 1.3 & 7.11 & 0.01 \\
\hline & Metastasis & Expression $\geq 4$ th quartile & $\leq$ 3rd quartile & 1.92 & 1.19 & 3.1 & 0.008 \\
\hline & & $\begin{array}{l}\text { Extranodal extension of } \\
\text { metastases }\end{array}$ & Without & 1.54 & 0.95 & 2.5 & 0.08 \\
\hline & & $\mathrm{pN} 2 / 3$ & pN1 & 1.43 & 0.73 & 2.77 & 0.3 \\
\hline & & $\mathrm{pT} 3 / 4$ & $\mathrm{pT} 1 / 2$ & 3.12 & 1.23 & 7.89 & 0.02 \\
\hline
\end{tabular}

Abbreviations: CI, confidence interval; HR, hazard ratio.

Significant $P$ values are indicated in bold. 
a

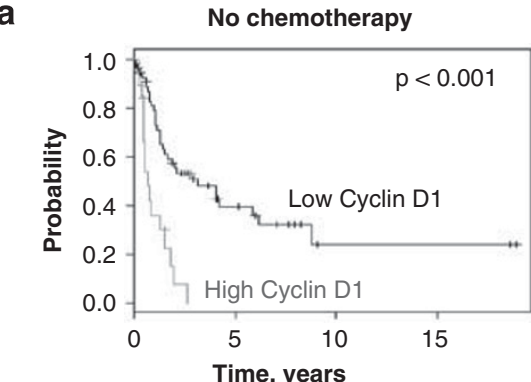

No. at risk

Low
Time, years

$\begin{array}{llll}60 & 12 & 2 & 2\end{array}$

b

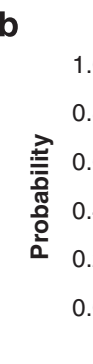

Cancer-specific survival

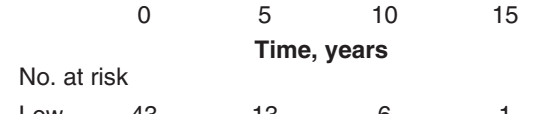

c

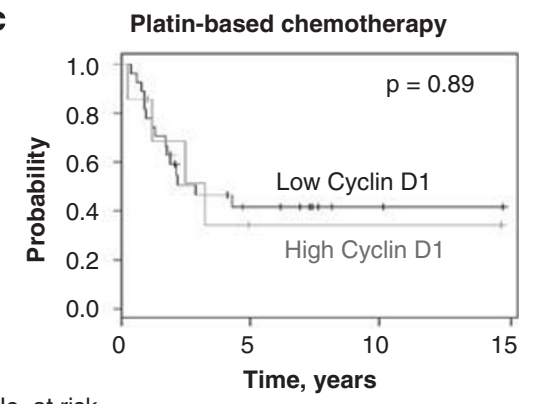

No. at risk

$\begin{array}{ll}\text { Low } & 27 \\ \text { High } & 7\end{array}$
8
2

1
1

Figure 5 Cancer-specific survival stratified according to the CyclinD1 expression in lymph node metastases. All patients without chemotherapy and with high expression die within 2.5 years after surgery (a). This prognostic impact of high CyclinD1 expression in the metastases is lost after any chemotherapy (b) and particularly after platin-based therapy (c).

seed metastases and to survive therapy. ${ }^{36,37}$ Knowledge about intra-tumor heterogeneity is important to assess the probability of a sampling error when evaluating prognostic and predictive biomarkers, and understanding of this heterogeneity is incomplete without the analysis of the metastasizing component, which can differ substantially from their primary tumor. ${ }^{38}$ In bladder cancer, CCND1 status was identical between tumor center and invasion front in $94 \%$ of the tested primary tumors (79\% non-amplified, $15 \%$ amplified) and different in $6 \%$. In the metastases, concordance between samples A and B was 97\% (76\% non-amplified; $22 \%$ homogeneously and $2 \%$ heterogeneously amplified). Finally, CCND1 amplification status between primary tumor and corresponding metastasis was also highly concordant; only one patient with an amplified primary tumor had a non-amplified metastasis and four patients showed an inverse genotype with no evidence of amplification in the primary tumors but amplified metastases. These data were generated from small cancer samples of $0.6 \mathrm{~mm}$ in diameter and defined clinically relevant subgroups. This suggests high intra-tumor homogeneity of CCND1 status in a given tumor and a minor sampling error in its determination.

The relation of CCND1 amplification status and CyclinD1 expression in bladder cancer has not been investigated yet. In all the tumor compartments (tumor center, invasion front, metastases), median CyclinD1 expression level in amplified samples was significantly higher compared with non-amplified samples. However, an amplified tumor sample could express the lowest CyclinD1 levels and vice versa non-amplified tumor samples could show the highest. The same phenomenon has also been observed in breast cancer $^{18}$ and indicates that overexpression of CyclinD1 in cancer is a complex mechanism in amplified cases also. This suggests that increased CCND1 gene copy numbers are multipliers of intracellular signals for protein expression but, on their own, not sufficient for CyclinD1 overexpression.

Amplification of $C C N D 1$ is an adverse prognostic factor in cancers of the breast, ${ }^{39}$ colon $^{39}$ and head and neck. ${ }^{40}$ In our bladder cancer cohort also, CCND1 amplification in either the primary tumor or the metastasis predicted poor outcome. Patients with this genetic defect doubled their probability of dying from bladder cancer compared with patients without this alteration, and this prognostic information was independent from the established risk factors. The high concordance of the CCND1 status between a primary tumor and its metastasis explains their aligned prognostic potential. However, the pathophysiological background for the aggressivity of amplified tumors is unclear and not necessarily mediated by CyclinD1. CCND1 amplification might also reflect a high degree of genetic instability, ${ }^{41}$ that by itself is associated with a particularly aggressive phenotype or, alternatively, other genes in the amplicon may contribute to malignancy. ${ }^{29}$ Contrarily to amplification status, CyclinD1 expression level in primary tumors was not strongly linked to the one of their metastases, and CyclinD1 overexpression was an adverse independent risk factor only in metastases. These differences in biomarker status and in its prognostic potential between both tumor components show that the primary tumor is not necessarily a surrogate for the metastasizing component. In general, the latter drives the disease and needs to be explored adequately to determine the relevance of prognostic and predictive molecules. Finally, the ability to stratify survival may differ between CCND1 amplification status and CyclinD1 expression level as previously reported for other cancers. ${ }^{18,42}$ Consequently, CCND1 and CyclinD1 are also no surrogates for each other, and investigations on genetic and protein level are necessary to determine the respective prognostic potential.

Bladder cancer, in general, has a limited chemosensitivity and better prediction of chemotherapeutic 
response in the individual tumor is highly warranted to personalize this therapy and avoid overtreatment. ${ }^{43}$ Interestingly, CyclinD1 has previously been reported to predict favorable response to chemotherapy in head and neck cancers. ${ }^{10,11}$ Most importantly, in our bladder cancer cohort also, CyclinD1 is a predictor of response to chemotherapy: while virtually all patients with high CyclinD1 expression in metastases and without chemotherapy die from cancer within 2.5 years after surgery, CSS significantly increases when adjuvant chemotherapy is given (5 year CSS: $22 \%$ ) and particularly after platin-based chemotherapy (5 year CSS: $37 \%$ ). No similar effect of chemotherapy was observed in the subgroup with low CyclinD1 expression. The successful chemotherapy treatment of the metastasizing tumor component with high CyclinD1 expression might help to personalize this application, and targeting CyclinD $1^{35}$ might supplement therapeutic options in such patients with reduced health condition not qualifying for conventional chemotherapy.

In conclusion, CCND1 amplification and CyclinD1 overexpression are independent adverse risk factors in metastasizing bladder cancer. Additionally, high CyclinD1 expression in the metastasizing component predicts favorable response to adjuvant chemotherapy. This information may help to individualize prognostication and administration of adjuvant therapies, thus improving patient management.

\section{Acknowledgements}

Received funding by Novartis Stiftung für medizinisch-biologische Forschung.

\section{Disclosure/conflict of interest}

The authors declare no conflict of interest.

\section{Disclaimer}

We confirm that the design and conduct of the work was performed by all of us. Manuscript has been written, read and approved by all of us. This work, or parts of it, have not been and will not be submitted elsewhere for publication.

\section{References}

1 Madersbacher S, Hochreiter W, Burkhard F, et al. Radical cystectomy for bladder cancer today-a homogeneous series without neoadjuvant therapy. J Clin Oncol 2003;21:690-696.

2 Stein JP, Lieskovsky G, Cote R, et al. Radical cystectomy in the treatment of invasive bladder cancer: longterm results in 1054 patients. J Clin Oncol 2001;19: 666-675.

3 Fleischmann A, Thalmann GN, Markwalder R, et al. Extracapsular extension of pelvic lymph node meta- stases from urothelial carcinoma of the bladder is an independent prognostic factor. J Clin Oncol 2005;23: 2358-2365.

4 Diehl JA. Cycling to cancer with cyclin D1. Cancer Biol Ther 2002;1:226-231.

5 Gillett C, Smith P, Gregory W, et al. Cyclin D1 and prognosis in human breast cancer. Int J Cancer 1996; 69:92-99.

6 Hui AM, Li X, Shi YZ, et al. Cyclin D1 overexpression is a critical event in gallbladder carcinogenesis and independently predicts decreased survival for patients with gallbladder carcinoma. Clin Cancer Res 2000;6: 4272-4277.

7 Jin M, Inoue S, Umemura T, et al. Cyclin D1, p16 and retinoblastoma gene product expression as a predictor for prognosis in non-small cell lung cancer at stages I and II. Lung Cancer 2001;34:207-218.

8 Pignataro L, Pruneri G, Carboni N, et al. Clinical relevance of cyclin D1 protein overexpression in laryngeal squamous cell carcinoma. J Clin Oncol 1998; 16:3069-3077.

$9 \mathrm{Yu} \mathrm{Z,} \mathrm{Weinberger} \mathrm{PM,} \mathrm{Haffty} \mathrm{BG,} \mathrm{et} \mathrm{al.} \mathrm{Cyclin} \mathrm{d1}$ is a valuable prognostic marker in oropharyngeal squamous cell carcinoma. Clin Cancer Res 2005;11: 1160-1166.

10 Akervall J, Kurnit DM, Adams M, et al. Overexpression of cyclin D1 correlates with sensitivity to cisplatin in squamous cell carcinoma cell lines of the head and neck. Acta Otolaryngol 2004;124:851-857.

11 Rodriguez-Pinilla M, Rodriguez-Peralto JL, Hitt R, et al. Cyclin A as a predictive factor for chemotherapy response in advanced head and neck cancer. Clin Cancer Res 2004;10:8486-8492.

12 Takeuchi H, Ozawa S, Ando N, et al. Cell-cycle regulators and the Ki-67 labeling index can predict the response to chemoradiotherapy and the survival of patients with locally advanced squamous cell carcinoma of the esophagus. Ann Surg Oncol 2003;10: 792-800.

13 Klein EA, Assoian RK. Transcriptional regulation of the cyclin D1 gene at a glance. J Cell Sci 2008;121: 3853-3857.

14 Kim JK, Diehl JA. Nuclear cyclin D1: an oncogenic driver in human cancer. J Cell Physiol 2009;220:292-296.

$15 \mathrm{Fu} \mathrm{M}$, Wang C, Li Z, et al. Minireview: Cyclin D1: normal and abnormal functions. Endocrinology 2004;145:5439-5447.

16 Hosokawa Y, Arnold A. Mechanism of cyclin D1 (CCND1, PRAD1) overexpression in human cancer cells: analysis of allele-specific expression. Genes Chromosomes Cancer 1998;22:66-71.

17 Cho EY, Choi YL, Han JJ, et al. Expression and amplification of Her2, EGFR and cyclin D1 in breast cancer: immunohistochemistry and chromogenic in situ hybridization. Pathol Int 2008;58:17-25.

18 Hadzisejdic I, Mustac E, Jonjic N, et al. Nuclear EGFR in ductal invasive breast cancer: correlation with cyclin-D1 and prognosis. Mod Pathol 2010;23:392-403.

19 Simpson JF, Quan DE, O’Malley F, et al. Amplification of CCND1 and expression of its protein product, cyclin D1, in ductal carcinoma in situ of the breast. Am J Pathol 1997;151:161-168.

20 Lopez-Beltran A, Luque RJ, Alvarez-Kindelan J, et al. Prognostic factors in stage T1 grade 3 bladder cancer survival: the role of G1-S modulators (p53, p21Waf1, p27kip1, Cyclin D1, and Cyclin D3) and proliferation index (ki67-MIB1). Eur Urol 2004;45:606-612. 
21 Shariat SF, Ashfaq R, Sagalowsky AI, et al. Correlation of cyclin D1 and E1 expression with bladder cancer presence, invasion, progression, and metastasis. Hum Pathol 2006;37:1568-1576.

22 Yurakh AO, Ramos D, Calabuig-Farinas S, et al. Molecular and immunohistochemical analysis of the prognostic value of cell-cycle regulators in urothelial neoplasms of the bladder. Eur Urol 2006;50:506-515.

23 Bringuier PP, Tamimi Y, Schuuring E, et al. Expression of cyclin D1 and EMS1 in bladder tumours; relationship with chromosome 11q13 amplification. Oncogene 1996;12:1747-1753.

24 Fristrup N, Birkenkamp-Demtroder K, Reinert T, et al. Multicenter validation of cyclin D1, MCM7, TRIM29, and UBE2C as prognostic protein markers in nonmuscle-invasive bladder cancer. Am J Pathol 2013;182: 339-349.

25 Lee CC, Yamamoto S, Morimura K, et al. Significance of cyclin D1 overexpression in transitional cell carcinomas of the urinary bladder and its correlation with histopathologic features. Cancer 1997;79:780-789.

26 Levidou G, Saetta AA, Karlou M, et al. D-type cyclins in superficial and muscle-invasive bladder urothelial carcinoma: correlation with clinicopathological data and prognostic significance. J Cancer Res Clin Oncol 2010;136:1563-1571.

27 Tut VM, Braithwaite KL, Angus B, et al. Cyclin D1 expression in transitional cell carcinoma of the bladder: correlation with p53, waf1, pRb and Ki67. Br J Cancer 2001;84:270-275.

28 Olsson H, Hultman P, Monsef N, et al. Immunohistochemical evaluation of cell cycle regulators: impact on predicting prognosis in stage $\mathrm{t} 1$ urinary bladder cancer. ISRN Urol 2012;379081.

29 Del Rey J, Prat E, Ponsa I, et al. Centrosome clustering and cyclin D1 gene amplification in double minutes are common events in chromosomal unstable bladder tumors. BMC Cancer 2010;10:280.

30 Zaharieva BM, Simon R, Diener PA, et al. Highthroughput tissue microarray analysis of 11q13 gene amplification (CCND1, FGF3, FGF4, EMS1) in urinary bladder cancer. J Pathol 2003;201:603-608.

31 Mills RD, Turner WH, Fleischmann A, et al. Pelvic lymph node metastases from bladder cancer: outcome in 83 patients after radical cystectomy and pelvic lymphadenectomy. J Urol 2001;166:19-23.

32 Studer UE, Danuser H, Merz VW, et al. Experience in 100 patients with an ileal low pressure bladder substitute combined with an afferent tubular isoperistaltic segment. J Urol 1995;154:49-56.

33 Sobin LH, Wittekind C (eds). TNM Atlas, 7th edn. Wiley-Lyss, Inc: New York, NY, USA; 2009; International Union Against Cancer.

34 Kononen J, Bubendorf L, Kallioniemi A, et al. Tissue microarrays for high-throughput molecular profiling of tumor specimens. Nat Med 1998;4:844-847.

35 Musgrove EA, Caldon CE, Barraclough J, et al. Cyclin D as a therapeutic target in cancer. Nat Rev Cancer 2011;11:558-572.

36 Marusyk A, Almendro V, Polyak K. Intra-tumour heterogeneity: a looking glass for cancer? Nat Rev Cancer 2012;12:323-334.

37 Marusyk A, Polyak K. Tumor heterogeneity: causes and consequences. Biochim Biophys Acta 2010;1805: 105-117.

38 Stoecklein NH, Klein CA. Genetic disparity between primary tumours, disseminated tumour cells, and manifest metastasis. Int J Cancer 2010;126:589-598.

39 Bahnassy AA, Zekri AR, El-Houssini S, et al. Cyclin A and cyclin D1 as significant prognostic markers in colorectal cancer patients. BMC Gastroenterol 2004; $4: 22$.

40 Myo K, Uzawa N, Miyamoto R, et al. Cyclin D1 gene numerical aberration is a predictive marker for occult cervical lymph node metastasis in TNM Stage I and II squamous cell carcinoma of the oral cavity. Cancer 2005;104:2709-2716.

41 Al-Kuraya K, Schraml P, Torhorst J, et al. Prognostic relevance of gene amplifications and coamplifications in breast cancer. Cancer Res 2004;64: 8534-8540.

42 Kaminagakura E, Werneck da Cunha I, Soares FA, et al. CCND1 amplification and protein overexpression in oral squamous cell carcinoma of young patients. Head Neck 2011;33:1413-1419.

43 Black PC, Agarwal PK, Dinney CP. Targeted therapies in bladder cancer-an update. Urol Oncol 2007;25: 433-438. 